\title{
Digital Watermarking Technology of Holographic Image Based on S-FFT Method
}

\author{
Li Wang, Fuping Liu *, Zirui Fu, Yukun Wang and Zhipeng Lu \\ ${ }^{*}$ Corresponding author
}

\begin{abstract}
In this paper, we use one fast Fourier algorithm of the Fresnel integral transform (S-FFT) to realize the digital watermarking information of hologram. The most important point is that by the four-step phase shifting method to obtain four holographic images. We can randomly choose a holographic image embedded in the vector image, thus realizing the digital watermarking technology of holographic images, and the remaining three holograms as watermarking information stored as public key in the optical code. For the host image of containing the watermarking information to do the inverse operation of the watermarking embedding process, we can extract the watermarking information hologram. Used the extracted watermarking hologram and others three public holograms we can reconstruct the watermarking image by four-step phase shifting hologram reconstruction. In this paper, the results of experiments are given by using MATLAB simulation software, the results show that under the premise which is almost without affecting the visual quality of the host image, the extracted image with high resolution, and the watermarking algorithm achieves the desired results.
\end{abstract}

Keywords-digital watermark; digital holography; four-step phase shifting; fresnel transform; fast convolution

\section{INTRODUCTION}

In the calculation method of digital holography, the Fresnel diffraction integral calculation method is commonly used [1]. Fresnel diffraction integral has a fast Fourier algorithm (S-FFT) [2] that has good stability in the case of changing the diffraction distance. And the domestic and foreign scholars in recent years have gradually begun to do research at the directions of digital holography and information hiding. Ibrahim COSKUN [3] proposed a new digital image steganography algorithm based on visible wavelength. Based on this technology, in 2015, Xia Kai-wei [4] with other workers put forward digital holographic watermarking algorithm and its implement with Fresnel transform. It not only has important theoretical significance but also has application prospect that scholars do research for digital holographic watermarking image embedding and extraction algorithm [5]. In order to reconstruct watermark image better and eliminate the inconvenience caused by the phase shift error, generalized phase shift algorithm developed gradually. Four-step phase-shifting algorithm is the most commonly algorithm used in phase shifting interferometry [6]. Compared with the multi-step phase-shifting algorithm, four-step phaseshifting algorithm has small phase shift steps; Compared with the two-step or three-step phase shift [7] algorithm, there is some redundancy information. It also has advantages like a strong anti-noise and high stability. In this paper, based on Fresnel diffraction integral of S-FFT algorithm we proposed the image information hiding and extraction algorithms by a four-step phase-shifting algorithm. Since the four-step phaseshifting algorithm can generate four watermark holograms, we choose one of them as confidential information and the rest as public information. Thus this form greatly enhances the security of watermark information.

\section{FRESNEL INTEGRAL FAST FOURIER ALGORITHM}

The formula of Fresnel diffraction integral for application is the most widely used, and it can be separately expressed as two forms of the Fourier transform and convolution. That is to say, there are two algorithms of fast Fourier transform and fast convolution algorithm. No matter what form, the most fundamental is the Fresnel diffraction integral, but we choose different forms in a different environment. For the Fresnel diffraction integral, we have

$$
\begin{gathered}
U(x, y)=\frac{\exp (j k z)}{j \lambda z} \exp \left[\frac{j k}{2 z}\left(x^{2}+y^{2}\right)\right] \int_{-\infty}^{\infty} \int_{-\infty}^{\infty}\left\{U_{0}\left(x_{0}, y_{0}\right)\right. \\
\left.\exp \left[\frac{j k}{2 z}\left(x_{0}^{2}+y_{0}^{2}\right)\right]\right\} \exp \left[-j \frac{2 \pi}{\lambda z}\left(x x_{0}+y y_{0}\right)\right] d x_{0} d y_{0}
\end{gathered}
$$

Where $U_{0}\left(x_{0}, y_{0}\right)$ and $U(x, y)$ are represented as complex amplitude of light the object plane and observation plane; $Z$ is the distance between the observation plane and the object plane; $\lambda$ is wavelength of light; $k=2 \pi / \lambda$ is wave number. That diffraction calculated the diffracted field distribution by the above formula is called Fresnel diffraction.

Formula (1) can be expressed as a fast Fourier transform FFT:

$$
\begin{gathered}
U(p \Delta x, q \Delta y)=\frac{\exp (j k z)}{j \lambda z} \exp \left[\frac{j k}{2 z}\left((p \Delta x)^{2}+(q \Delta y)^{2}\right)\right] \\
\times F F T\left\{U_{0}\left(m \Delta x_{0}, n \Delta y_{0}\right) \exp \left[\frac{j k}{2 z}\left(\left(m \Delta x_{0}\right)^{2}+\left(m \Delta y_{0}\right)^{2}\right)\right]\right\}_{\frac{p \Delta x}{\lambda d}, \frac{q \Delta y}{\lambda d}} \\
(p, q, m, n=-N / 2,-N / 2+1, \cdots, N / 2-1)
\end{gathered}
$$

In the formula, $\Delta x=\Delta y$ is calculated by the fast Fourier transform corresponding to the spatial sampling interval. As 
we can be seen from the above, the Fresnel diffraction process is equivalent process that the object plane wave field passes through linear space-invariant system. The formula (2) will be the source of the program we need to conduct MATLAB simulation.

\section{FOUR-STEP PHASE-SHIFTING ALGORITHM}

The basic principle of the phase shift method is that the reference arm of the interferometer by phase shifter regularly to move the reference mirror, and the optical path difference between the reference beam and the test beam is changed, then the position of the stripes moves accordingly. So making the light intensity at any point in the interference field is cosine transform, that we can use the photosensitive electronic element CCD to collect three or more pieces of interference patterns of different phases. Consequently, zero-order image and conjugate image interference can be removed. The coherent superposition of reference light and object wave occurred in the CCD, the interference field intensity distribution recorded by CCD:

$$
\begin{gathered}
I(x, y, \theta)=\left|R_{0}(x, y) \exp (j \theta)+O_{0}(x, y) \exp (j \varphi)\right|^{2} \\
=\left|R_{0}\right|^{2}+\left|O_{0}\right|^{2}+R_{0} O_{0} \exp (-j \varphi) \exp (j \theta)+R_{0} O_{0} \exp (-j \theta) \exp (j \varphi)
\end{gathered}
$$

Where $\theta$ is the introduction of a stepped phase shift angle; $R$ and $O$ are plane waves and the amplitude of the scattered light reaches the recording medium of the CCD; $\varphi$ is the angle between the original and $Z$ axis.

Four-step phase-shifting algorithm refers to the process of recording a hologram for a state of the object under test using the phase shifter of the reference light to take four different phase shift value. Typically, four figures of the intensity distribution can be obtained by formula. (3)

$$
\left\{\begin{array}{l}
I(x, y, 0)=\left|R_{0}\right|^{2}+\left|O_{0}\right|^{2}+2 R_{0} O_{0} \cos \varphi \\
I\left(x, y, \frac{\pi}{2}\right)=\left|R_{0}\right|^{2}+\left|O_{0}\right|^{2}+2 R_{0} O_{0} \sin \varphi \\
I(x, y, \pi)=\left|R_{0}\right|^{2}+\left|O_{0}\right|^{2}-2 R_{0} O_{0} \cos \varphi \\
I\left(x, y, \frac{3 \pi}{2}\right)=\left|R_{0}\right|^{2}+\left|O_{0}\right|^{2}-2 R_{0} O_{0} \sin \varphi
\end{array}\right.
$$

Using four-step phase-shifting algorithm is available to the complex amplitude distribution of object waves on CCD:

$$
O(x, y)=\frac{1}{4 R}\left\{[I(x, y, 0)-I(x, y, \pi)]+j\left[I\left(x, y, \frac{\pi}{2}\right)-I\left(x, y, \frac{3 \pi}{2}\right)\right]\right\}
$$

The formula (5) is used to reconstruct digital holographic image. This method can eliminate the zero-order image and conjugate image, improve the signal to noise ratio of digital hologram, and improve the quality of the reconstructed image.

\section{INFORMATION HIDING AND EXTRACTION ALGORITHM}

Digital watermarking technology is an information hiding technique. In this paper, the secret information (watermarking information) is picture made by myself. After doing Fresnel diffraction integral with four-step phase-shifting, obtained four holographic images that one of this will be embedded into a vector image provided by ourselves, we call this secret information after transforming as digital watermarking.

Embedded image is a holographic image $(H 1)$ generated by four-step phase-shifting method of the watermarking information. In the spatial domain $H 1$ will be loaded on the vector image, so that we can achieve a digital watermark embedding process. The formula is expressed as:

$$
W=C+a H 1
$$

where: $a$ is the scale factor for controlling the intensity of the watermark image; $C(\theta, \varphi)$ represents vector image.

And the information containing digital watermarking is the information of $W$ obtained in the formula (6). And the original image is actually a vector image also shown as $C$. The extraction algorithm is very simple, that is the inverse of the formula (6):

$$
H 1^{\prime}=(W-C) / a
$$

where: $H 1^{\prime}$ is the extracted holographic image.

\section{ANALYSIS OF RESUlTS}

\section{A. Generated Holographic Images}

We select a logo $(178 \times 177 \times 3$ bit $)$ of Beijing Institute of Graphic Communication as the original. Setting the appropriate number of sampling points $\mathrm{N}=256$, diffraction distance $\mathrm{z}=1000$ and optical wavelength $\lambda=600 \mathrm{~nm}$ as the simulation conditions, we can add random noise on the original phase information, and discrete and adjust the size of the original image. After a series of processing, it can obtain watermarking image (object plane image) $(256 \times 256 \mathrm{bit})$ in this experiment. Using of formula (1), we make S-FFT operation for watermark image to obtain the object light reached the CCD field amplitude. Above all, it is made full preparations for generating holographic images. Finally, we generate four holograms (H1, H2, H3, H4) used equation (4). The simulation results were shown below. As the four images are similar $(0, \pi / 2,3 \pi / 2, \pi$-phase), the remaining phase of the three pictures are not displayed, and in this paper only shows the $\pi / 2$-phase picture. 


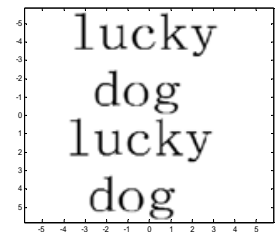

FIGURE I. WATERMARKING IMAGE

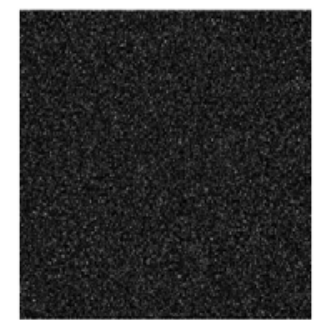

FIGURE II. HOLOGRAM H1 (PHASE: $\frac{\pi}{2}$ )

\section{B. Hide and Extract Holographic Image}

To select randomly as a secret information a digital watermark from the four holograms [here selecting the hologram H1 (256 ×256bit) ], we choose the figure of Lena $(256 \times 256 \times 3$ bit $)$ for the host image to embed watermarking information by Eq.(6), realized the information hiding of holograms image, the host image of contained watermarking information was shown in Figure IV, From figure IV and figure III we can find that the two image are almost nodifference, holographic image embedded without changing the visual quality of host image .

For the watermarked host image to carry out embedding inverse transform we can extract holographic image from the embedded holographic image by Eq. (7).

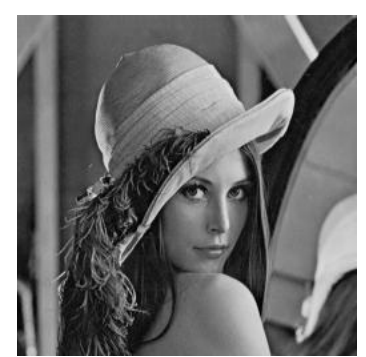

FIGURE III. HOST IMAGE

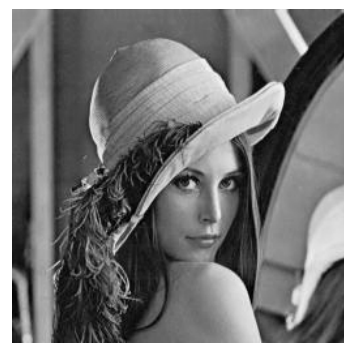

FIGURE IV. HOST IMAGE OF CONTAINED WATERMARKING

\section{Reconstruction of Holographic Image}

According to the four-step phase-shifting algorithm principle, the extracted secret holographic image has been reconstructed. Next complex amplitude of the light field was reconstructed by Eq. (5). And then applying inverse transformation of Fresnel diffraction integral form (S-FFT), we can reconstruct the original watermarking, information image by Four-step phase-shifting algorithm ( $H 1$, see Figure VI). We only applied the remaining 3 holographic images to reconstruct watermarking image was shown as Figure $\mathrm{V}$ (here the absence of $H 1$ ). So $H 1$ can serve as key information in transmission process, which is secret information.

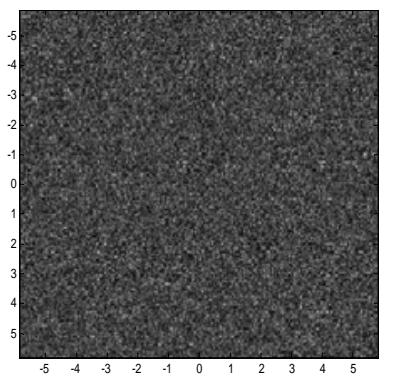

FIGURE V. RECONSTRUCTED WATERMARKING IMAGE (USING 3 HOLOGRAPHIC IMAGES)

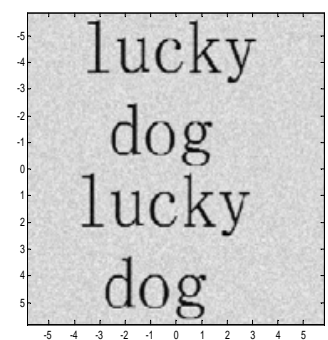

FIGURE VI. RECONSTRUCTED WATERMARKING IMAGE (USING 4 HOLOGRAPHIC IMAGES)

\section{Analysis}

Compared Figure III with Figure IV, we can see that this algorithm does not alter the quality of the host image substantially. So the method in this paper can hide our secret information better. By comparing Figure I with Figure VI, we can find that the four-step phase-shifting algorithm can completely restore the watermarking image. But if we are missing one of the secret information (holographic image), we will not get the watermarking information. That is to say that we put one of the holographic images as a key hidden in the vector image to ensure the safety and reliability of the information transmission.

\section{CONCLUSION}

Our algorithm overcomes the problems that watermarking image extracted by other algorithms is vague. Through the experiments we also found that in the case, at the absence of any one of four holographic images, the watermark image 
cannot be reconstructed. So choosing any of them as the secret information from the holographic images is a high security, and it would be greatly increase the difficulty of crack. These are the advantages that four-step phase-shifting algorithm is applied to the information hiding, and it also is the core of the algorithm. Currently we have not found that the digital watermarking algorithm which combined the information encrypted and a hidden with the four-step phase shift method. Our experimental results show this method not only has a good information hiding effect, but also has a high clarity that the watermarking information is extracted from the host image by this method, it is a kind of good method that has application prospects.

\section{ACKNOWLEDGMENT}

This work was supported by The Beijing City Board of Education Science and technology key project (KZ201510015015), The College Student Research Program of 2016, Beijing City Board of Education Science and technology project (KM201510015009), Natural Science Foundation of Beijing (4142016) and PXM2016_014223_000025.

\section{REFERENCES}

[1] Tomoyoshi Shimobab, Nobuyuki Masuda, Tomoyoshi Ito. Arbitrary shape surface Fresnel diffraction.OPTICS EXPRESS, 2012, 20 (8).

[2] Arash Sabatyan,Leila Elahi.FFT-based convolution algorithm for fast and precise numerical evaluating diffracted field by photon sieve.Optik,2013,(124):4960-4962.

[3] Ibrahim COSKUN, Feyzi AKAR, Ozdemir CETIN. A new digital image steganography algorithm based on visible wavelength. Turkish Journal of Electrical Engineering \&Computer Science, 2013,(21):548564.

[4] XIA Kai-wei, LIU Fu-ping, etc. Digital Holographic Watermarking Algorithm and Its Implementation Based on Fresnel Transform [J]. Journal of Beijing Institute of Graphic Communication.2015,23(2).

[5] Zhang Ru-yu. Research of Image Watermarking Information Hiding Technology Algorithm [D]. South China University of Technology. 2014.

[6] ZHANG Qian, XU Xian-feng, etc. Phase-shift Extraction and Wave Reconstruction in Four-step Phase-shifting Interferometry [J]. OptoElectronic Engineering, 2011, 38(8);

[7] Zhang Shuquan,Zhou Jianyang.A new estimation method for two-steponly quadrature phase- 